\title{
Localization of human recombinant adenoviral vectors to the mitochondria following transduction of human cell lines
}

\author{
B. J. MORRISON ${ }^{1}$, M. ABU-ASAB ${ }^{2}$, J. C. MORRIS ${ }^{1}$, J. C. STEEL $L^{1,3}$
}

\begin{abstract}
${ }^{1}$ Division of Hematology-Oncology, University of Cincinnati Cancer Institute, University of Cincinnati, Cincinnati, Ohio, 45267, USA; ${ }^{2}$ Section of Histopathology, National Institutes of Health, Bethesda, MD, 20892, USA; ${ }^{3}$ School of Health, Medical and Applied Sciences, Central Queensland University, Queensland, Australia
\end{abstract}

\begin{abstract}
Summary. - Recently, mitochondria have been shown to have a vital role in the innate immune response to viral infection. In response, viruses such as adenovirus, have developed mechanisms to alter mitochondrial function through direct or indirect interaction with the mitochondria. The interaction of human recombinant adenoviral vectors directly with human mitochondria has not previously been shown. We demonstrate that human recombinant adenoviral vectors co-localize to mitochondria. We show that the adenoviral vectors are present within the membranes of the mitochondria and that they cause ultrastructural changes to the cristae. Further, we show that the adenoviral genome is also present in intact mitochondria. We have posited that the interaction between the adenovirus and the mitochondria may act to inhibit mitochondrial function. We have also posited that the transport of the adenoviral genome to the mitochondria may allow the future use of this vector as a tool for gene therapy of mitochondrial diseases.
\end{abstract}

Keywords: mitochondria; human recombinant adenovirus; gene therapy; viral vectors; mitochondrial DNA; electron microscopy

\section{Introduction}

Mitochondria are double membrane encapsulated cellular organelles containing their own genome consisting of a circular double-stranded 16,569 base pair long molecule coding for 37 genes (13 polypeptides) involved in oxidative phosphorylation and respiration (Taanman, 1999). The mitochondrial DNA (mtDNA) has frequent mutations due to inefficient DNA repair system processes (Croteau and Bohr, 1997). mtDNA is also more susceptible to oxidative damage than nuclear DNA (nDNA). Mutations in mtDNA have been shown to induce a number of diseases such as Kearns-Sayre

E-mail: brian.morrison.uc@gmail.com; phone: +1-(301)-319-9957. Abbreviations. Ad pVII = adenoviral core protein pVII; mtDNA $=$ mitochondrial DNA; MLS = mitochondrial localization signal; $\mathrm{MOI}=$ multiplicities of infection; $\mathrm{nDNA}=$ nuclear DNA; $\mathrm{PFU}=$ plaque forming units; $\mathrm{rAd}=$ recombinant adenoviral vectors; $\mathrm{qPCR}=$ quantitative PCR syndrome, Mitochondrial myopathy, encephalopathy, lactic acidosis, and stroke (MELAS) syndrome, and Leber's hereditary optic neuropathy (Taylor and Turnbull, 2005). The prevalence of mitochondrial diseases is estimated to be 1 in every 5,000 to 10,000 live births (Haas et al., 2007; Schaefer et al., 2008). Mitochondrial dysfunction is also believed to play a role in neurodegenerative diseases, aging, and cancer (de Moura et al., 2010). Approaches to specifically target mitochondria have implications for both cancer therapy (Morrison et al., 2009) and gene therapy (Kyriakouli et al., 2008).

Mitochondria have a number of vital cellular functions including cellular respiration, calcium storage and signaling, and the control of apoptosis - programmed cell death. Recent studies have also shown that mitochondria play a major role in anti-viral host defense mechanisms (Koshiba, 2013). Some viruses, such as adenovirus, have adapted to thwart this defense by producing proteins that directly alter mitochondrial function (Anand et al., 2014).

Adenoviruses are non-enveloped double-stranded DNA viruses with the ability to infect a wide variety of dividing 
and non-dividing cells. The adenoviral virion is composed of a protein capsid with 240 hexon components and 12 pentons, and a nucleoprotein core that contains the viral DNA and internal proteins. The adenoviral genome encodes early-expressed proteins required for DNA replication (E1A, E1B, E2, E3 and E4), late structural proteins (L1, L2, L3, L4 and L5), and intermediate-expressed proteins (IX and IVa2), flanked by inverted terminal repeats (McConnell and Imperiale, 2004). In addition to their function in DNA replication, adenoviral proteins E1A and E1B have been shown to localize to the mitochondria and can alter mitochondrial-induced apoptosis. Mitochondrial localization of human recombinant adenoviral vectors ( $\mathrm{rAd}$ ) deficient in E1 and E3 genes has also been previously shown in bovine and murine cells, with some evidence of disrupting mitochondrial integrity (Alesci et al., 2007). To date, adenoviral infection of human mitochondria has not been shown.

In this study, we examine the ability of rAd to localize to the mitochondria of a number of human cell lines, and describe evidence of morphological changes in the mitochondria following transduction.

\section{Materials and Methods}

Adenoviral vectors. Ad.null, an E1-, E3-deleted rAd were generated by homologous recombination with the AdMax system (Microbix, Toronto, Canada). The resulting adenovirus was double plaque-isolated, expanded on 293 cells, purified on a cesium chloride gradient, titered by serial dilution as plaque forming unit(s) $(\mathrm{PFU}) / \mathrm{ml}$ and stored at $-80^{\circ} \mathrm{C}$ as previously described (Sakai $e t$ al., 2004).

Cancer cell lines. Human cancer cell lines A549, HepG2, and HeLa (American Type Culture Collection (ATCC), Manassas, VA), and HUH-7 (GMP-Creative Biolabs, Shirley, NY) were cultured as adherent cells in RPMI-1640 (Mediatech, Inc., Manassas, VA) supplemented with $10 \%$ heat-inactivated fetal calf serum (Invitrogen, Carlsbad, CA) and 1\% penicillin G-streptomycin (Invitrogen). Briefly, each cell line was seeded onto 6 -well plates at $1 \times 10^{6}$ cells/ well (Thermo Fisher Scientific, Waltham, MA).

Fluorescent labeling of adenovirus particles. Fluorescent labeling of rAd vector particles was performed using a Cy5 labeling kit (Abcam, Cambridge, MA) using the manufacturer's protocol. Briefly, modifier reagent was added to aliquots of $1 \times 10^{12}$ vector particles in suspension in $0.05 \mathrm{M}$ HEPES buffer $\mathrm{pH}$ 7.2. The modified viral particles were then added to Cy5 conjugate mix and incubated for 30 min in the dark at room temperature. The reaction was stopped by adding Cy5 quencher reagent. Excess unreacted dye was eliminated by dialysis against PBS.

Photomicrographs of cells following adenoviral transduction. A549, HepG2, HeLa, and HUH-7 cells grown on coverslips were used for photomicrograph assessment of adenovirus and mitochondrial interaction. Briefly, cells were transduced with Cy5-labeled adenovirus at multiplicity of infection (MOI) of $300 \mathrm{PFU} / \mathrm{cell}$. The cells were incubated for $6 \mathrm{~h}$ and washed. Mitochondria were stained using MitoTracker ${ }^{\circledR}$ Green FM probe (Invitrogen) according to the manufacturer's instructions. Cells were then fixed with $3.7 \%$ formaldehyde in complete growth medium at $37^{\circ} \mathrm{C}$ for 15 min. Cell nucleuses were stained with PureBlu ${ }^{\mathrm{TM}}$ Hoechst 33342 Nuclear Staining Dye (Bio-Rad, Hercules, CA) according to the manufacturer's instructions. Cells were then rinsed $1 \mathrm{x}$ with PBS and assessed for fluorescence (Hoechst maximum emission at $461 \mathrm{~nm}$, MitoTracker ${ }^{\bullet}$ Green FM at $516 \mathrm{~nm}$, and Cy5 at $643 \mathrm{~nm}$ ) using an Olympus BX53 upright fluorescent microscope (Olympus, Tokyo, Japan).

Electron micrographs of cells following adenoviral transduction. HUH-7 cells, grown on 6-well dishes were transduced with Adnull at an MOI of 300 and incubated for one hour. The cells were then washed with PBS, fixed in $2.5 \%$ glutaraldehyde for $1 \mathrm{~h}$, and harvested by scraping and centrifugation at $1000 \mathrm{x} g$ for $10 \mathrm{~min}$. Cells were double-fixed in osmium tetroxide (0.5\%), dehydrated, and embedded into Spurr's epoxy resin. Ultrathin sections $(90 \mathrm{~nm})$ were cut, double-stained with uranyl acetate and lead citrate, and examined with a Philips CM10 transmission electron microscope (Electronic Instruments, Mahwah, N.J., USA).

Detection of adenoviral genomes in the mitochondria.HUH-7, HepG2 and A549 cells were used to assess whether adenoviral DNA was present in the intact mitochondria following transduction with rAd. Cells grown in a $75 \mathrm{~cm}^{2}$ flask were transduced with Ad-null at an MOI of 300 and incubated at $37^{\circ} \mathrm{C}$ for $6 \mathrm{~h}$. The cells were washed with PBS and the mitochondria isolated using Qproteome Mitochondria Isolation Kit (Qiagen, Germantown, MD) according to the manufacturer's instructions. Isolated mitochondria were treated with proteinase $\mathrm{K}$ and DNase I ( 5 ug proteinase K, 0.4 ug DNase I in $20 \mathrm{mmol} / \mathrm{l} \mathrm{Tris-HCl}, 20 \mathrm{mmol} / \mathrm{l} \mathrm{MgCl}, 500 \mathrm{mmol} / \mathrm{l} \mathrm{KCl} \mathrm{buffer}$ ) to remove any genomic or viral contaminants. The mitochondria were washed and pelleted $3 \mathrm{x}$ before resuspension in $20 \mathrm{mmol} / \mathrm{l}$ EDTA and heated at $70^{\circ} \mathrm{C}$ for $15 \mathrm{~min}$ to inactivate DNase. Total DNA was extracted from mitochondria using a DNeasy extraction kit (Qiagen Inc. Valencia, CA). All DNA extracted was quantified by spectrophotometer (A260/280) (NanoDrop 2000, Thermo Fisher Scientific, Waltham, MA). The purity of the preps was examined by assessing the presence of mtDNA and nDNA by quantitative PCR (qPCR) using the following primers and probes: MtDNA: Forward (mt2981): 5'-ACGACCTCGATGTTGAATC-3', Reverse (mt3245): 5'-GCTCTGCCATCTTAACAAACC-3', Probe (mtDNA)5'-TTCAGACCGGAGTAATCCAGGTCG-3' (6FAM labeled 5', TAMRA quencher 3') and nDNA: 3 Actin Forward: 5'-CTGCCT GACGGCCAGG-3', ß Actin Reverse: 5'-GGAAAAGAGCCT CAGGGCAT-3', Probe (B Actin)-5'-CATCACTATTGGCAAC GAGCGGTTCC-3' (6FAM labeled 5', TAMRA quencher 3'). qPCR was used to measure the amount of adenovirus in cellular mitochondria, cytosol, and nucleus as previously described (Steel et al., 2007). A quantitative Ad5 real-time PCR assay was performed using the following primers and probe (Applied Biosystems): Ad5 33414 fwd 5'-GTAATTCACCACCTCCCGGTA-3', Ad5 33562 rev 

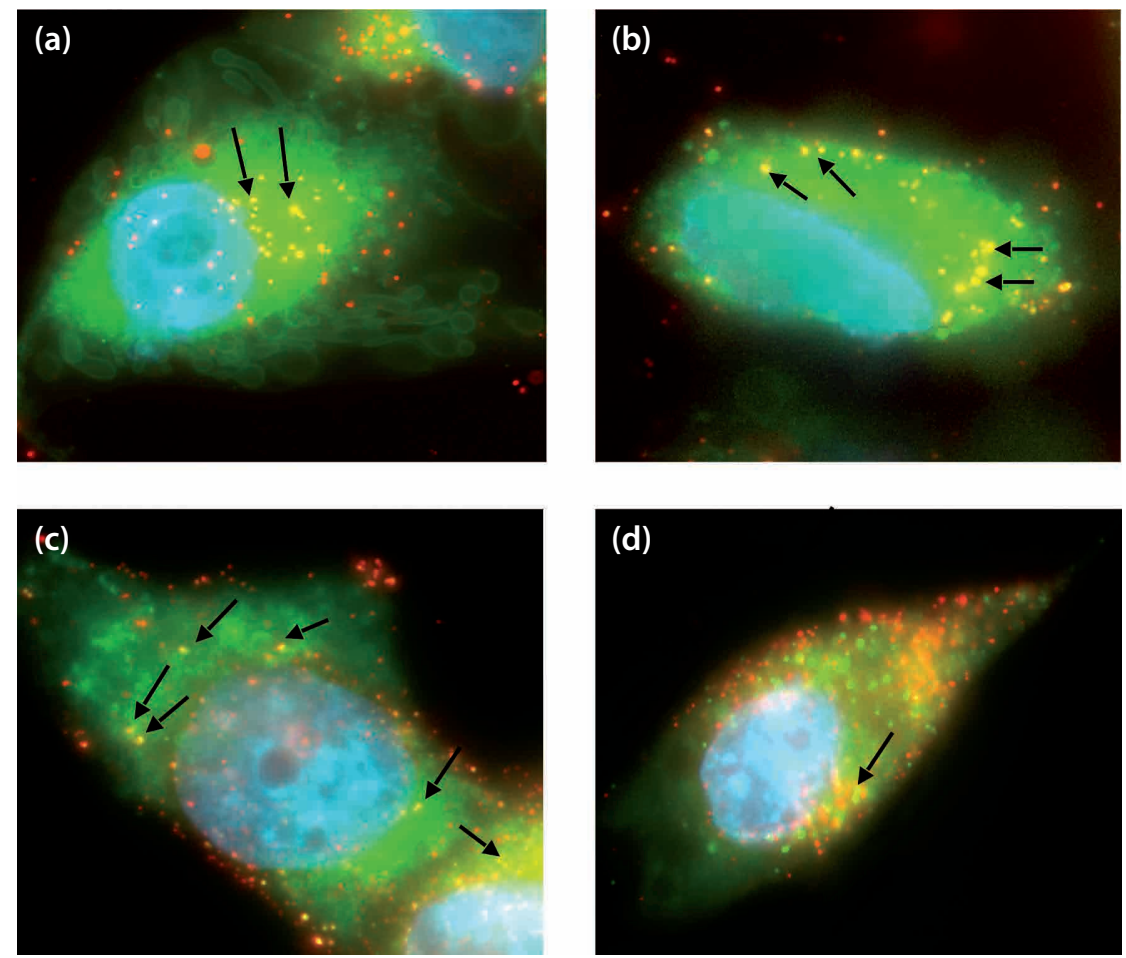

Fig. 1

Human recombinant adenovirus localizes to mitochondria in human cells. Photomicrographs of human cell lines (a) HepG2, (b) A549, (c) HUH-7, and (d) HeLa

Mitochondria are shown in green, adenovirus in red, and blue shows nuclear staining. Areas of co-localization of adenovirus and mitochondria appear yellow. Black arrows point out areas of co-localization.

5'-GGCTCTCCACTGTCATTGTTC-3', Ad5 probe 5'-ACCTCT GATTAAACATGGCGCCATCC-3' (FAM labeled 5', TAMRA quencher $\left.3^{\prime}\right)$. DNA was amplified using $100 \mathrm{nmol} / \mathrm{l}$ of each primer and $200 \mathrm{nmol} / \mathrm{l}$ of the probe using the following cycling conditions: $2 \mathrm{~min}$ at $50^{\circ} \mathrm{C}, 10 \mathrm{~min}$ at $95^{\circ} \mathrm{C}, 45$ cycles of $15 \mathrm{~s}$ at $95^{\circ} \mathrm{C}$ (denaturation) and 45 cycles of $1 \mathrm{~min}$ at $63^{\circ} \mathrm{C}$ (extension/annealing) in an ABI 7700 DNA sequencer (Applied Biosystems). A standard curve was generated using pre-titered adenovirus standards ranging from $10^{0}$ to $10^{6} \mathrm{PFU}$ to determine copy number. PCR reactions for each sample were performed in triplicate using the same oligonucleotide primers and fluorescent probe, reaction conditions, and cycling.

Statistics. Statistical analysis was preformed using Student's ttest using SigmaStat version 3.1 statistical software (Systat, Point Richmond, CA). Data is presented as mean \pm standard deviation.

\section{Results}

Localization of human recombinant adenovirus with mitochondria occurs within human cancer cell lines

In order to examine whether rAd co-localizes with cellular mitochondria we fluorescently labeled the adenoviral capsid with Cy5 and transduced (A) HepG2, (B) A549, (C) HUH-7, and (D) HeLa cells (Fig. 1). We co-stained the mitochondria with MitoTracker ${ }^{\circledast}$ Green and the nucleus with Hoechst 33342 nuclear staining dye. In all four cell lines, we could visualize viral particles (red staining) and mitochondria (green staining). The majority of viral particles were located on the surface of the cells and within the nucleus, however, distinct co-localization of capsid with mitochondria was seen in each of the cell lines, as indicated by yellow staining. No cytopathic effect was noted in cells following infection with rAd for up to seven days (data not shown).

Human recombinant adenovirus is present at mitochondria one hour following infection

In order to examine how the rAd co-localized with the mitochondria, we transduced HUH-7 cells with adenovirus and examined the cells by transmission electron microscopy (Fig. 2). Multiple distinct rAd particles were found both inside the mitochondria and at the mitochondrial surface of infected cells. In some cells, multiple viral particles were seen within single mitochondria. Interestingly, those mitochondria with viral particles within them showed 


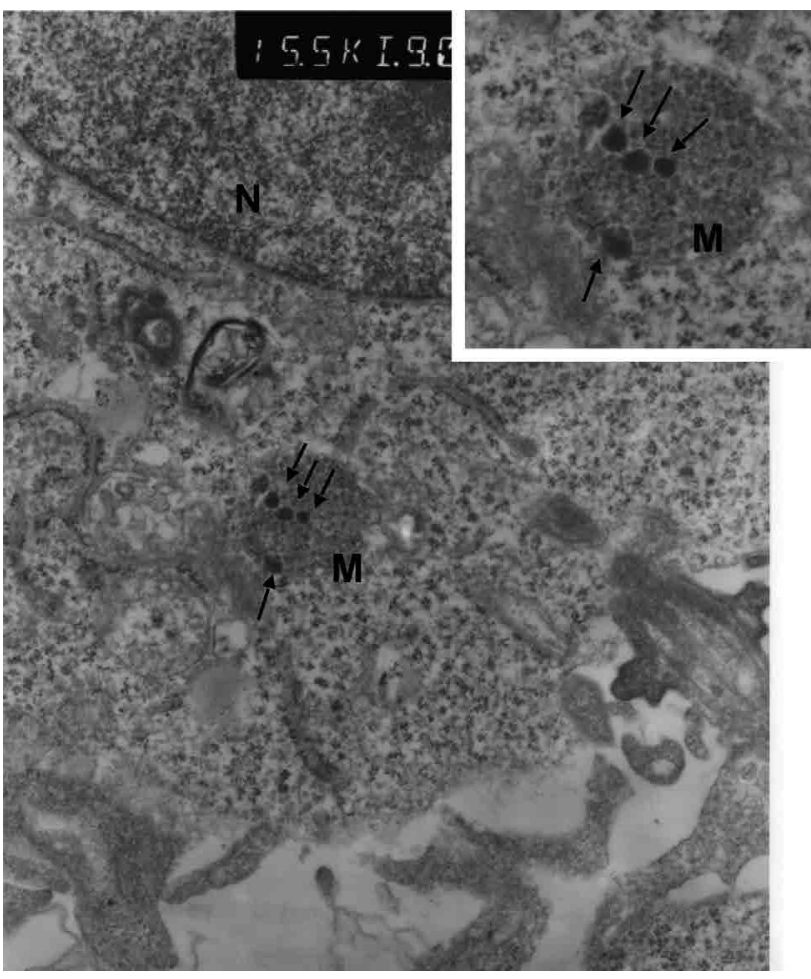

Fig. 2

Human recombinant adenovirus present in the mitochondria of human cells

Electron micrographs taken one hour after infection with recombinant human adenovirus. Mitochondria (M) shows the presence of adenovirus localization (black arrows). Nucleus (N).

ultrastructural deterioration with breakdown of internal membrane structures.

Human recombinant adenoviral genomes are detected within membrane-bound mitochondria

Next, we examined whether the adenoviral particles detected within the mitochondria contained the viral genome and whether the mitochondria retained external membrane integrity. To test this, we isolated mitochondria from HepG2, HUH-7 and A549 cells transduced with rAd vectors, treated the mitochondrial fraction with proteinase $\mathrm{K}$ and DNase I to remove any contaminating DNA and to test membrane integrity, and assayed adenoviral genomes by qPCR (Fig. 3). The results showed that all three cell lines had detectable levels of rAd DNA within the mitochondrial fraction. In this Proteinase K/DNase I-treated mitochondrial fraction we also showed that mtDNA could be detected and that nDNA was not, indicating that membrane integrity was retained.

\section{Discussion}

The complex interplay between mitochondria and viruses has developed through a long process of pathogen-host coevolution. Recent evidence points to the mitochondria in playing an important role in innate immunity and antiviral immunity (West et al., 2011; Koshiba, 2013). In this interplay, viruses, including adenovirus, have developed a number of strategies to overcome the defense mechanisms of the host cells. Recently, influenza A virus protein PB1-F2 has been shown to translocate into the inner membrane space of mitochondria and directly impair innate immunity (Yoshizumi et al., 2014). One innate mechanism that cells have developed to prevent viral infection is the induction of apoptosis, mediated by the mitochondria. The adenoviral E1 proteins have been shown to have an important role in the induction of mitochondrial-induced apoptosis in response to adenoviral infection (Rao et al., 1992). Adenoviral E1A has been shown to stabilize p53 following infection, while E1B proteins have been shown to inhibit the function of p53 (Debbas and White, 1993). Interestingly, the E1B-19K protein has been shown to localize within the mitochondria and interact with p53 to inhibit apoptosis (Lomonosova et al., 2005).

In this study, using human rAd deficient in both E1 and E3 genes, we showed with fluorescent and transmission election microscopy that the vectors without $\mathrm{E} 1$ genes were able to co-localize with mitochondria in human cells. These results in human cells were similar to previous findings by Alesci $e t$ al. (2007), who found adenoviral virions present in bovine adrenal chromaffin cells following rAd transduction. In line with this previous study, we also found evidence for internal mitochondrial membrane degeneration following adenoviral

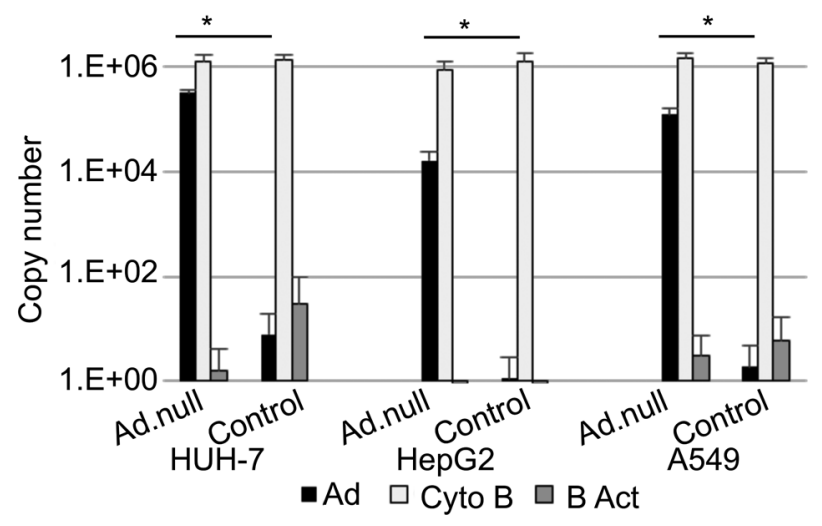

Fig. 3

Human recombinant adenovirus can be detected in the mitochondria Mitochondria from HUH-7, HepG2 and A549 cells were assayed for the presence of adenoviral DNA, mitochondrial DNA (Cyto B) or Genomic DNA (B Actin) following transduction with adenoviral vectors (Ad.null) or PBS (control). Error bars are standard deviation. ${ }^{*} P<0.001$. 
co-localization. The ultrastructural degeneration of internal mitochondrial membranes and the breakdown of cristae has been reported for other viral infections, such as vaccinia (Morgan et al., 1954), tobacco mosaic virus (Weintraub and Ragetli, 1964), and western equine encephalomyelitis virus (Morgan et al., 1961), and may represent a biological process to alter the function of the mitochondria.

Building on the finding that human rAd co-localizes to mitochondria in human cells, we also showed that adenoviral DNA was present in mitochondria following viral transduction. It has previously been shown that adenovirus can be misdirected inside infected cells (Wang et al., 2013). Interestingly, the adenoviral DNA was detected encapsulated within the outer membrane-intact mitochondria as evidenced by its initial resistance to DNase and proteinase $\mathrm{K}$. While this is the first time that adenoviral DNA has been detected in mitochondria, Anand et al. previously showed that the adenoviral core protein pVII (Ad pVII) contains a mitochondrial localization signal (MLS) and this protein can be found in the mitochondria following adenoviral infection/ transduction (Anand et al., 2014). The adenoviral genome within the virion is closely associated with Ad pVII protein, which condenses the viral DNA into a nucleosome-like structure (Vayda et al., 1983). The Ad pVII protein remains associated with the viral DNA during infection, only being released during early gene transcription (Chen et al., 2007). Given the close interaction of Ad pVII with the viral DNA, and its MLS, it is possible that Ad pVII is responsible for the localization of the adenoviral genome within the mitochondria. The presence of Ad pVII in the mitochondria has been shown to have a number of biological functions that may benefit the adenovirus, including the induction of an increase in adenosine triphosphate, maintaining mitochondrial membrane potential and inhibiting apoptosis (Anand et al., 2014).

In this study we have provided evidence that human recombinant adenoviral vectors can co-localize with mitochondria in human cells. We further demonstrate that the adenovirus is able to enter the mitochondria. The entry into the mitochondria appears to disrupt the internal cristae but does not appear to disrupt the outer mitochondrial membrane. We also show for the first time that adenoviral DNA is transported to the mitochondria following transduction/infection, opening up the potential for recombinant adenoviral vectors to be used in future mitochondrial gene therapy approaches.

\section{References}

Alesci S, Abu-Asab M, Perera SM, Tsokos M, Morris JC, Pacak K (2007): Mitochondrial localization of human recombinant adenovirus: from evolution to gene therapy.
Neuroimmunomodulation 14, 221-223. https://doi. org $/ 10.1159 / 000113065$

Anand SK, Gaba A, Singh J, Tikoo SK (2014): Bovine adenovirus 3 core protein precursor $\mathrm{pVII}$ localizes to mitochondria, and modulates ATP synthesis, mitochondrial $\mathrm{Ca} 2+$ and mitochondrial membrane potential. J. Gen. Virol. 95, 442-452. https://doi.org/10.1099/vir.0.057059-0

Chen J, Morral N, Engel DA (2007): Transcription releases protein VII from adenovirus chromatin. Virology 369, 411-422. https://doi.org/10.1016/j.virol.2007.08.012

Croteau DL, Bohr VA (1997): Repair of oxidative damage to nuclear and mitochondrial DNA in mammalian cells. J. Biol. Chem. 272, 25409-25412. https://doi.org/10.1074/ jbc.272.41.25409

de Moura MB, dos Santos LS, Van Houten B (2010): Mitochondrial dysfunction in neurodegenerative diseases and cancer. Environmental and molecular mutagenesis 51, 391-405. https://doi.org/10.1002/em.20575

Debbas M, White E (1993): Wild-type p53 mediates apoptosis by E1A, which is inhibited by E1B. Genes Dev. 7, 546-554. https://doi.org/10.1101/gad.7.4.546

Haas RH, Parikh S, Falk MJ, Saneto RP, Wolf NI, Darin N, Cohen BH (2007): Mitochondrial disease: a practical approach for primary care physicians. Pediatrics 120, 1326-1333. https://doi.org/10.1542/peds.2007-0391

Koshiba T (2013): Mitochondrial-mediated antiviral immunity. Biochim. Biophys. Acta 1833, 225-232. https://doi. org/10.1016/j.bbamcr.2012.03.005

Kyriakouli DS, Boesch P, Taylor RW, Lightowlers RN (2008): Progress and prospects: gene therapy for mitochondrial DNA disease. Gene Ther. 15, 1017-1023. https://doi. org/10.1038/gt.2008.91

Lomonosova E, Subramanian T, Chinnadurai G (2005): Mitochondrial localization of p53 during adenovirus infection and regulation of its activity by E1B-19K. Oncogene 24, 6796-6808. https://doi.org/10.1038/sj.onc. 1208836

McConnell MJ, Imperiale MJ (2004): Biology of adenovirus and its use as a vector for gene therapy. Hum. Gene Ther. 15, 1022-1033. https://doi.org/10.1089/hum.2004.15.1022

Morgan C, Ellison SA, Rose HM, Moore DH (1954): Structure and development of viruses observed in the electron microscope. II. Vaccinia and fowl pox viruses. J. Exp. Med. 100, 301-310. https://doi.org/10.1084/jem.100.3.301

Morgan C, Howe C, Rose HM (1961): Structure and development of viruses as observed in the electron microscope. V. Western equine encephalomyelitis virus. J. Exp. Med. 113, 219-234. https://doi.org/10.1084/jem.113.1.219

Morrison BJ, Andera L, Reynolds BA, Ralph SJ, Neuzil J (2009): Future use of mitocans against tumour-initiating cells? Mol. Nutr. Food Res. 53, 147-153. https://doi.org/10.1002/ mnfr.200800254

Rao L, Debbas M, Sabbatini P, Hockenbery D, Korsmeyer S, White E (1992): The adenovirus E1A proteins induce apoptosis, which is inhibited by the E1B $19-\mathrm{kDa}$ and Bcl-2 proteins. Proc. Natl. Acad. Sci. USA 89, 7742-7746. https://doi. org/10.1073/pnas.89.16.7742 
Sakai Y, Morrison BJ, Burke JD, Park JM, Terabe M, Janik JE, Forni G, Berzofsky JA, Morris JC (2004): Vaccination by genetically modified dendritic cells expressing a truncated neu oncogene prevents development of breast cancer in transgenic mice. Cancer Res. 64, 8022-8028. https://doi. org/10.1158/0008-5472.CAN-03-3442

Schaefer AM, McFarland R, Blakely EL, He L, Whittaker RG, Taylor RW, Chinnery PF, Turnbull DM (2008): Prevalence of mitochondrial DNA disease in adults. Ann. Neurol. 63, 35-39. https://doi.org/10.1002/ana.21217

Steel JC, Morrison BJ, Mannan P, Abu-Asab MS, Wildner O, Miles BK, Yim KC, Ramanan V, Prince GA, Morris JC (2007): Immunocompetent syngeneic cotton rat tumor models for the assessment of replication-competent oncolytic adenovirus. Virology 369, 131-142. https://doi. org/10.1016/j.virol.2007.07.022

Taanman JW (1999): The mitochondrial genome: structure, transcription, translation and replication. Biochim. Biophys. Acta 1410, 103-123. https://doi.org/10.1016/S0005$\underline{2728(98) 00161-3}$

Taylor RW, Turnbull DM (2005): Mitochondrial DNA mutations in human disease. Nature reviews. Genetics 6, 389-402. https://doi.org/10.1038/nrg1606
Vayda ME, Rogers AE, Flint SJ (1983): The structure of nucleoprotein cores released from adenovirions. Nucleic Acids Res. 11, 441-460. https://doi.org/10.1093/nar/11.2.441

Wang IH, Suomalainen M, Andriasyan V, Kilcher S, Mercer J, Neef A, Luedtke NW, Greber UF (2013): Tracking viral genomes in host cells at single-molecule resolution. Cell Host Microbe 14, 468-480. https://doi.org/10.1016/j. chom.2013.09.004

Weintraub M, Ragetli HW (1964): An Electron Microscope Study of Tobacco Mosaic Virus Lesions in Nicotiana Glutinosa L. J. Cell Biol. 23, 499-509. https://doi.org/10.1083/ jcb.23.3.499

West AP, Shadel GS, Ghosh S (2011): Mitochondria in innate immune responses. Nature reviews. Immunology 11, 389-402. https://doi.org/10.1038/nri2975

Yoshizumi T, Ichinohe T, Sasaki O, Otera H, Kawabata S, Mihara K, Koshiba T (2014): Influenza A virus protein PB1-F2 translocates into mitochondria via Tom 40 channels and impairs innate immunity. Nat. Commun. 5, 4713. https:// doi.org/10.1038/ncomms5713 